\title{
Survey on an Efficient Coverage and Connectivity of Wireless Sensor Networks using Intelligent Algorithms.
}

\author{
Dr.M.Siddappa \\ Professor \& Head, Dept. of Computer Science \& Engg. \\ Sri Siddhartha Institute of Technology, Tumkur, India \\ Email:siddappa.p@gmail.com \\ Channakrishna raju \\ Research Scholar, Sri Siddhartha University, Tumkur, India \\ Email:ckrajussit@gmail.com
}

\begin{abstract}
Wireless sensor networks are often deployed for data-gathering or monitoring in a geographical region. This paper explains an important issue to maintain the fidelity of the sensed data while minimizing energy usage in the network. Nature inspired computation like evolutionary computation, swarm intelligence etc., which offers practical advantages to the researcher facing difficult optimization problems. The genetic algorithms are used for efficient connectivity and coverage. Single Objective Genetic Algorithms (SOGA) method is used to yield good results in terms of Coverage, but the objective's graph had shown Pareto optimal designs with differing Endurance. However it is attractive to offer Pareto optimal designs to a user willing to settle for a poorer Coverage in order to gain in Endurance, so that the sensor network lasts longer. This explains concept of Multiple Objective Genetic Algorithm (MOGA) and its implementation and results which are compared to those of the SOGA. Endurance and Robustness to deployment inaccuracy tend to work in the same direction. A MOGA was conducted with the Coverage and Robustness as objectives. The main objective of this paper is to propose new Strength Perito Evolutionary Algorithm (SPEA) method along with clustering, this will reduce the distances between the sensor nodes that increase the efficiency of the nodes and also increase the connectivity. This will increase lifetime of sensors and connectivity.
\end{abstract}

Index Terms - Connectivity and Coverage, SOGA, MOGA, SPEA, Clustering.

\section{Introduction}

Recent advances in hardware miniaturization, communication technologies, and low-cost mass production have facilitated the emergence of wireless sensor networks (WSNs) that consist of small, inexpensive, battery-powered, and wirelessly connected sensors. The WSNs have brought up various new applications, including surveillance ${ }^{[1-2]}$ home security, and environmental monitoring ${ }^{[3-4]}$ WSN sensors are deployed randomly or systematically to collect information about their surroundings within their sensing range.

A promising WSN application is long-term surveillance in hostile or distant environments. Using WSNs for military surveillance, for example, involves deploying numerous sensors throughout the region of interest by aircraft to detect enemy activity or equipment. However, a key consideration in the design of WSNs is the power supply since replacing batteries in sensors is often impractical. Although a considerable number of studies have addressed energy efficiency issues in generic wireless ad hoc networks, distributed sensing applications impose new constraints on sensor network coverage ${ }^{[5] \text {. }}$

The problem of finding the maximum number of covers to extend WSN lifetime has been modeled as the SET K-COVER problem ${ }^{[6]}$. Provided K covers, the lifetime of WSNs can ideally be extended by a factor of $\mathrm{K}$ using the above approach under the coverage constraint. The SET K-COVER problem has been proven to be NP-complete. Under the assumption NP $\neq$ $\mathrm{P}$, no exact algorithm can solve this problem in polynomial time. Some heuristic algorithms have been presented, but they generally suffer from the trade-off between solution quality and running time. Recently, proposed using genetic algorithm (GA) to deal with this trade-off ${ }^{[7]}$. The GA achieves near-optimal solutions in acceptable time but requires information on the value of $\mathrm{K}$ or its upper bound, which is usually unobtainable. Additionally, such approaches rarely yield optimal solutions.

Soga ${ }^{[8]}$ is used for evaluate the efficiency. The selection is based on elitism, where the $\mathrm{N}$ individuals with highest Coverage are passed on to the new generation. Its disadvantage is its tendency to produce a homogenized population early, with often suboptimal results.

The draw back of SOGA is overcome in MOGA ${ }^{[8]}$. The GA itself is identical than the one used in the single objective case, with the exception of the selection, which must take into account both objectives. Since the goal of the MOGA is to provide a uniformly 
populated $\mathrm{PF}$, the weighted sum approach was rejected since it assumes an a priori knowledge of the user's preference of one objective over the other. Several schemes were devised to incorporate both objectives in the selection, and as in the case for the SOGA the deterministic elitist selection outperformed Binary Tournament and Roulette Wheel Selection.

These intelligent algorithms are ubiquitous now a days, having been success-fully applied to numerous problems from different domains, including optimization, automatic programming, machine learning, operations research, bioinformatics, and social systems. The objective of this paper is:

○ Propose new Method for increasing the coverage and connectivity of wireless sensor networks using genetic algorithms.

- Propose new SPEA method along with clustering, this will reduce the distances between the sensor nodes that increase the efficiency of the nodes and also increase the connectivity.

\section{Related Work}

The many aspects of the WSN lifetime problem include sensor activity scheduling [6,9] network structure [5], data aggregation [10-11], and routing protocol ${ }^{[12-13] .}$ (For a complete survey, see ${ }^{[1]}$ ). This study focuses on sensor activity scheduling. The problem of extending WSN lifetime by sensor activity scheduling was first modeled as the SET K-COVER problem by Slijep, cevic and Potkonjak ${ }^{[2] . ~ T h e y ~ p r o v e d ~}$ the NP-completeness of this problem by reduction from the minimum cover problem ${ }^{[14]}$. To solve this problem, the authors proposed the most constrained minimally constraining covering heuristic (MCMCC). This approach runs in polynomial time but often yields unsatisfactory results. Cardei and $\mathrm{Du}^{[9]}$ formulated WSN lifetime extension as the disjoint set covers (DSC) problem, which is analogous to the SET K-COVER problem.

Cardei and $\mathrm{Du}^{[9]}$ presented a heuristic algorithm, called maximum covers using mixed integer programming (MCMIP), to solve the DSC problem. Although the MCMIP method can find the optimal solution, its implicit exhaustive search requires exponential running time. The above approaches for extending WSN lifetime, however, suffer from the trade-off between solution quality and running time. For the SET K-COVER problem, the MCMCC takes only polynomial time but often yields unsatisfactory solutions. On the other hand, the MCMIP ensures optimal solutions but at the cost of exponential time complexity. To address this issue, Damin ${ }^{[8]}$ designed an integer-coded GA in which a generates $\mathrm{Ci} \in \mathrm{N}$ indicates the group number assigned to sensor $\mathrm{Si}$, and a chromosome represents the group arrangement of all sensors for covers. In the course of evolution, the groups gradually form covers, i.e., they satisfy the constraint of full coverage. Studies have demonstrated that this algorithm outperforms MCMCC in terms of the number of covers and is much faster than MCMIP. Nevertheless, one defect is that, owing to the integer representation of chromosomes, this GA requires an upper bound on the number of covers, which is usually unobtainable. Moreover, like most other GAs, the proposed GA rarely yields optimal solutions. Therefore, an algorithm is still needed to consistently deliver, within an acceptable running time, good activity schedules for extending WSN lifetime.The several methods are used for increases the coverage and connectivity of wireless sensor networks and these methods re discussed below:

SET K-COVER Problem: ${ }^{[6]}$ The problem is to organize mutually exclusive sensor nodes into a number of covers or sets each of which can fully cover the monitoring area A. The activity duration for each cover is the same and thus the energy consumption is uniform among nodes. As the lifetime of the network is direct proportional with the number of allocated covers, the goal is to maximize the number of covers.

Low Power Coverage: ${ }^{[15]}$ ILP1 formulations for the Minimum0-1 Cover, Minimal Cover with Sensor Field Intensity and Balanced Operation Scheduling problems. The Minimum 0-1 Cover tries to find the minimum number of sensors that can cover the entire monitoring area A while the Minimal Cover with Sensor Field Intensity tries to achieve the same goal with the guarantee that the sensor field intensity for each region of $\mathrm{A}$ is above a user specified threshold value.

Target Coverage: ${ }^{[5]}$ An efficient target coverage mechanism for sensor networks. The idea is to extend the network lifetime by organizing sensors into the maximal number of set covers. These set covers are activated successively such that at any given time only a set is active. The nodes from the active set will be in the active state while all the others will be in the sleep state. A key Difference between this approach ${ }^{[1]}$ and is that the sensor nodes can participate in multiple sets (the covers do not contain mutually exclusive nodes). The single restriction is that the sum of all time weights associated with the sets a node belongs to has to be 1 . Another difference is that this approach covers a set of targets, not an area as we have seen in previous works. They formalize the Maximum Set Covers (MSC) problem and they prove that is NP-complete.

Preserving Coverage: ${ }^{[16]} \mathrm{A}$ distributed and localized node scheduling algorithm capable to minimize the number of active nodes while preserving area coverage. The scheduling runs in rounds. Each round starts with a self-scheduling phase in which each node investigate its off-duty eligibility. Specifically, if a node's sensing range is sufficiently covered by its neighbors is eligible to turn off and save energy

CCP: CCP or Coverage Configuration Protocol ${ }^{[17]}$ is one of the first solutions that tries to achieve both 
coverage and connectivity in a single protocol. CCP decides if a sensor node has to be active or not according with the actual coverage of its sensing area. If its sensing area is already sufficiently covered by the neighboring nodes, the node can be Inactive and enter in the SLEEP mode. A node can be in there states: SLEEP, LISTEN and ACTIVE. In the SLEEP mode a node sleeps to conserve energy. In ACTIVE state the node actively senses the environment and communicates with other sensors. CCP ensures connectivity for this particular case, however sparse connectivity is ensured in the middle of the network.

PEAS: PEAS ${ }^{[18]}$ uses a similar technique for turning off nodes. However, PEAS design targets a much more hostile environment where: i) node failures are high, ii) the node deployment density is high and iii) Due to hardware limitations, sensor nodes cannot run complex protocols. PEAS protocol is simple and consists of two algorithms: Probing Environment, which determines which nodes should work and Adaptive Sleeping, which determines how to adjust dynamically the sensors' sleep times in order to keep a constant wakeup rate. At the beginning all the nodes are sleeping for a exponentially distributed random time. When a nodes wakes-up, it broadcasts a PROBE message within a certain range $\mathrm{Rp}$. Any working node that receives the message responds with a REPLY. There are no relationships between sensing and communication ranges specified, nor strong coverage or connectivity guarantees

OGDC: The Optimal Geographical Density Control algorithm is another solution which minimize the number of active sensors used to conserve energy while ensuring coverage and connectivity preservation in high density sensor networks. A similar theorem ${ }^{[10]}$ to simplify the integrated connected coverage problem. In addition, they also devise several optimality conditions for covering a region A with the minimum overlap of the covered neighbor areas.OGDC that a reduced number of deployed nodes are required to achieve coverage. OGDC uses rounds and thus require time synchronization among nodes. OGDC tries to optimize the set of active nodes based on their relative location (optimality conditions) whenever possible.

\section{Model Implementation}

The genetic algorithms are used to obtain the better performance than the other approaches. So we are trying to design a model with genetic algorithm to get efficient connectivity and coverage for wireless sensor networks

\subsection{Genetic Algorithms;}

Genetic Algorithms are adaptive heuristic search algorithms ${ }^{[19]}$. It was introduced as a computational analogy of adaptive systems. They are based on the principles of the evolution via natural selection, employing a population of individuals that undergo selection in the presence of variation-inducing operators such as mutation and crossover. To evaluate individuals a fitness function is used and reproductive success varies with fitness.

A Genetic Algorithms operates through a simple cycle of stages:

- Generate of a "population" of strings,

- $\quad$ Evaluate of each string,

- Selection of best strings and

- Genetic manipulation to create new population of strings.

The Genetic Algorithm cycle is presented as shown in the fig. 1

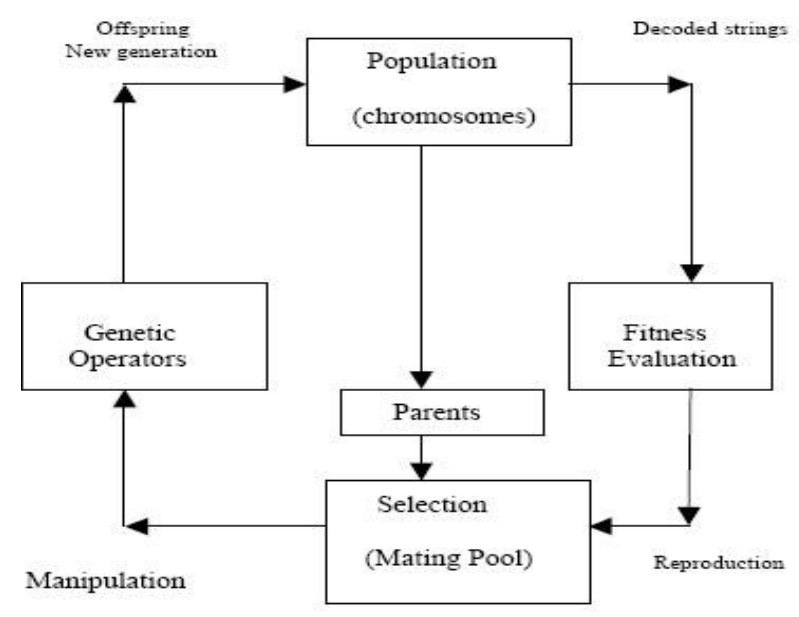

Fig: 1 : Genetic Algorithm Cycle

A Genetic Algorithm performs fitness tests on new structures to select the best population. Fitness determines the quality of the individual on the basis of the defined criteria. In nature, an individual's fitness is its ability to pass on its genetic material. Anything that contributes to this ability contributes to the organism's overall fitness. This ability includes traits that enable it to survive and further reproduce.

The genetic algorithms has the following parameter should be consider for design of model.

1. Population: A population consists of a group of individuals called chromosomes that represent a complete solution to a defined problem. Each chromosome is a sequence of 0 s or $1 \mathrm{~s}$. The initial set of the population is a randomly generated set of individuals. A new population is generated by two methods: steady-state GA and generational GA.

2. Fitness: In a GA, fitness is evaluated by the function defining the problem. The fate of an individual chromosome depends on the fitness value. The chances of survival are higher for better fitness values. 
3. Selection: The selection process determines which of the chromosomes from the current population will mate (crossover) to create new chromosomes. These new chromosomes join the existing population.

4. Crossover: Crossover is also known as recombination of component materials due to mating. It is a simulation of the sexual reproductive process which is responsible for the transfer of genetic inheritance.

5. Mutation: As a result of crossover, the new generation introduced will only have the traits of the parents. This can sometimes lead to a problem where no new genetic material is introduced in the offspring. Mutation allows new genetic patterns to be introduced in the new chromosomes. Mutation introduces a new sequence of generations into a chromosome but there is no guarantee that mutation will produce desirable features in the new chromosome

\subsection{SOGA (Single Objective Genetic Algorithm)}

Soga ${ }^{[8]}$ is used for evaluate the efficiency. The selection is based on elitism, where the $\mathrm{N}$ individuals with highest Coverage are passed on to the new generation. Its disadvantage is its tendency to produce a homogenized population early, with often suboptimal results. A steady improvement in Coverage can be noticed. As expected the Endurance is declining as the SOGA progresses, because networks with good coverage have poor endurance. SOGA yielded good results in terms of Coverage with less Endurance. However it is attractive to offer Pareto optimal designs to a user willing to settle for a poorer Coverage in order to gain in Endurance, so that the sensor network lasts longer. This possibility is not offered by the SOGA.

\subsection{MOGA (Multiple Objectives Genetic Algorithm).}

The draw back of SOGA is overcome in MOGA ${ }^{[8]}$. The GA itself is identical than the one used in the single objective case, with the exception of the selection, which must take into account both objectives. Since the goal of the MOGA is to provide a uniformly populated $\mathrm{PF}$, the weighted sum approach was rejected since it assumes an a priori knowledge of the user's preference of one objective over the other. Several schemes were devised to incorporate both objectives in the selection, and as in the case for the SOGA the deterministic elitist selection outperformed Binary Tournament and Roulette Wheel Selection. The pool of individuals is then sorted from the best ranked (nondominated individuals) to the worst ranked. Deterministic selection then keeps the $\mathrm{N}$ best individuals. This selection scheme insures that the current Pareto best networks are kept from generations to generations, irrespective of their objectives value. This makes it possible to keep a uniformly populated
PF. It has been said earlier that the number of sensors considered was fixed beforehand to 5. The study was conducted assuming all sensors had ranges RS and RCOMM equal to 2 . This was done by running the MOGA for increasing values of number of sensors, up to 15 . Fig. 2 shows the plot of the Coverage and of the Endurance versus the number of sensors.

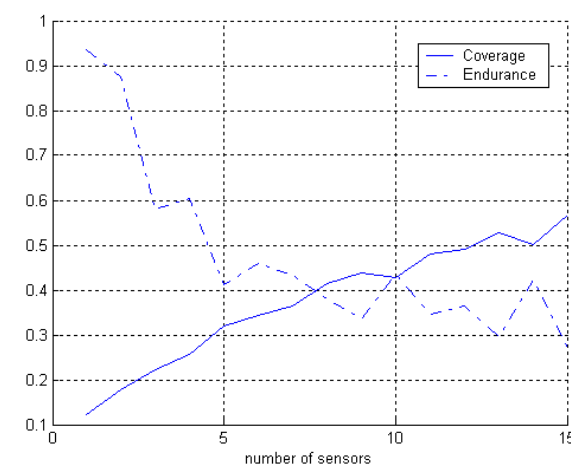

Fig. 2 : The plot of the Coverage and of the Endurance versus the number of sensors.

If the number of sensors is increased beyond 15 , it should be expected that the Coverage converges to 1 and that the Endurance stops decreasing and instead increases, as more sensors serve as relays. For a number of sensors lower than 15 the Endurance decreases with the number of sensors. Using this graph the user can determine what value of Coverage and Endurance can be expected from the MOGA, and accordingly choose the number of sensors to be placed. MOGA with elitist selection, which yielded good results in terms of PF population. This framework can then be implemented in a more realistic model for the communication and sensing of the sensors.

MOGA was the first multi-objective GA that explicitly used Pareto-based ranking and niching techniques together to encourage the search toward the true Pareto front while maintaining diversity in the population. Therefore, it is a good example to demonstrate how Pareto based Ranking and fitness sharing can be integrated in a multi-objective GA. The procedure of the MOGA ${ }^{[20]}$ is given as follows:

\section{Procedure MOGA}

Step 1: Start with a random initial population $\mathrm{P}_{0}$. Set $\mathrm{t}=0$.

Step 2: If the stopping criterion is satisfied, return $\mathrm{P}_{\mathrm{t}}$.

Step 3: Evaluate fitness of the population as follows

Step 3.1 Assign a rank $r(x, t)$ to each solution $x \in P_{t}$ using the ranking scheme using $r 2_{(x, t)}=1+n q(x, t)$

Step 3.2: Assign a fitness values to each solution based on the solution's rank as follows: 


$$
f(x, t)=\sum_{k=1}^{r[u k j]-1} n k-.5 x(n r(n, t)-1)
$$

Where $\mathrm{n}_{\mathrm{k}}$ is the number of solutions with rank $\mathrm{k}$

Step 3.3: calculate the niche count $\mathrm{nc}(\mathrm{x}, \mathrm{t})$ of each solution based $\mathrm{x} \in \mathrm{Pt}$ using

Step 3.4: calculate the shared fitness value of each solution $\mathrm{x} \in \mathrm{Pt}$ as follows:

$$
f^{\prime}(x, t)=f(x, t) / n c(x, t) .
$$

Step 3.5: Normalize the fitness values by using the shared fitness values

$$
\begin{gathered}
f^{\prime \prime}(x, t)=\frac{F 1[x, t) n r[x, t]}{\sum f 1(x, t)} f(x, t) \\
y \in \operatorname{Pt} r(y, t)=r(x, t)
\end{gathered}
$$

Step 4: Use a stochastic selection method based on 11 $\mathrm{f}^{11}$ to select parents for the mating pool. Apply crossover and mutation on the mating pool until offspring population

$\mathrm{Q}_{\mathrm{t}}$ of size $\mathrm{N}$ is filled. Set $\mathrm{Pt}+1=\mathrm{Q}_{\mathrm{t}}$.

Step 5: Set $\mathrm{t}=\mathrm{t}+1$, go to Step 2 .

The performance of MOGA is implemented by improving the elitist selection using SPEA 2

\subsection{Elitism}

Elitism in the context of single-objective GA means that the best solution found so far during the search always. Survives to the next generation. In this respect, all non dominated solutions discovered by a multi-objective GA are considered elite solutions. However, implementation of elitism number of possible elitist solutions. Early multi-objective GA did not use elitism. However, most recent multi-objective GA and their variations use elitism. As discussed in ${ }^{[20-}$ 22], multi-objective GA using elitist strategies tend to outperform their non-elitist counterparts. Multiobjective GA uses two strategies to implement elitism

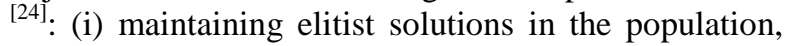
and (ii) storing elitist solutions in an external secondary list and reintroducing them to the population.

\subsection{Proposed new clustering with SPEA}

When an external list is used to store elitist solutions, several issues must be addressed. The first issue is which solutions are going to be stored in elitist list E. Most multi objective GA store non-dominated solutions identified so far during the search ${ }^{[23]}$, and $\mathrm{E}$ is updated each time a new solution is created by removing elitist solutions dominated by a new solution or adding the new solution if it is not dominated by any existing elitist solution. This is a computationally expensive operation. Several data structures have been proposed to efficiently store, update, and search in list $\mathrm{E}$.Another issue is the size of list E. Since there might possibly exist a very large number of Pareto optimal solutions for a problem, the elitist list can grow extremely large. Therefore, pruning techniques have been proposed to control the size of E. For example, SPEA uses the average linkage clustering method ${ }^{[24]}$ to reduce the size of $\mathrm{E}$ to an upper limit $\mathrm{N}$ when the number of the non-dominated solutions exceeds $\mathrm{N}$ as follows:

Step 1: Initially, assign each solution $\mathrm{x} \in \mathrm{E}$ to a cluster ci,

$\mathrm{C}=\{\mathrm{c} 1 ; \mathrm{c} 2 ; \ldots ; \mathrm{cM}\}$

Step 2: Calculate the distance between all pairs of clusters ci and cj as follows:

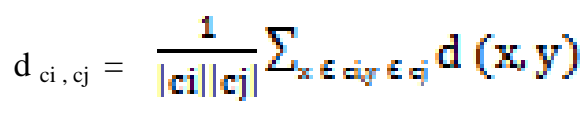

Here, the distance $d(x, y)$ can be calculated in objective function space.

Step 3: Merge the cluster pair ci and cj with the minimum distance among all clusters into a new cluster.

Step 4: If $|\mathrm{C}|<\mathrm{N}$, go to Step 5, else go to Step 2.

Step 5: For each cluster, determine a solution with the Minimum average distance to all other solutions in the same cluster (called the centroid solution). Keep the Centroid solutions for every cluster and remove other solutions from $\mathrm{E}$.

Figure 3 shows the method of cluster formation after doing the genetic algorithm. In this figure, the sink is shown at the middle of the Environment with a black square. The regular nodes are shown with black circles and cluster heads are shown by the white circles which are bigger than the normal nodes 


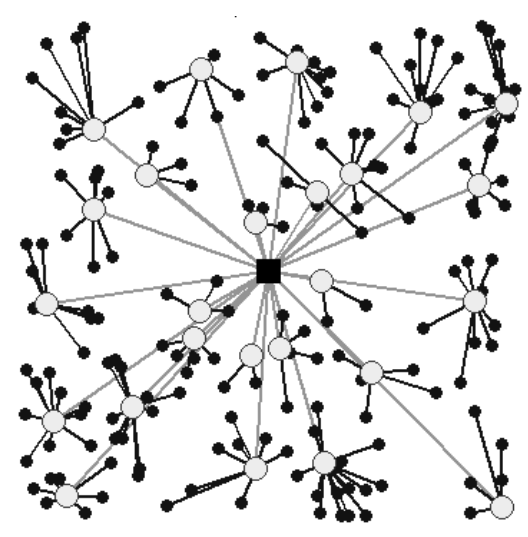

Fig 3: cluster formation

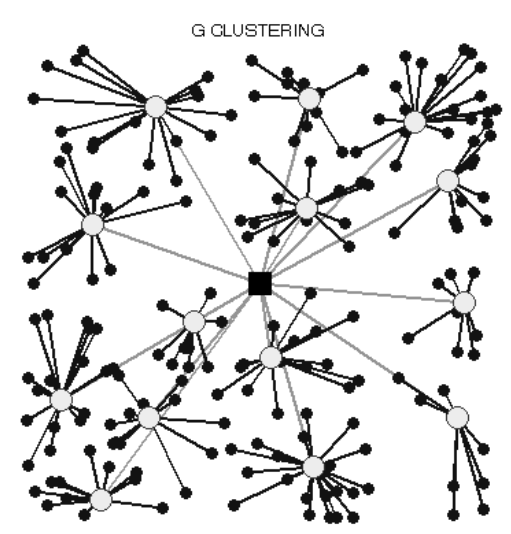

Fig: 4 cluster formation with SPEAalong with clustering

Figure 4 shows the method of the formation of clusters after doing SPEA with Clustering. In this figure, the sink is shown at the middle of the environment with a black square. The regular nodes are shown with black circles and cluster heads are shown by the white circles which are bigger than the normal nodes.

In fig. 3 shows that number edges coming towards the sink is large that will increase the network traffic and the distance between the nodes, so the coverage is not efficient. To overcome this draw back we propose the SPEA along with clustering as shown in fig 4 . This reduces the distance and network traffic between the nodes thus increases the connectivity.

\section{Conclusion}

Biology has fascinating facts influencing the area of computer science. Nature-inspired Computations have already achieved remarkable success. This paper surveyed several solutions for coverage ands connectivity in wireless sensor networks using nature inspired algorithms (genetic algorithm). As we have already seen early work focused only on the coverage problem. The widely adopted solution is to schedule nodes into sleep state whenever possible while ensuring coverage preservation. Most of these works do not consider different coverage degrees and adopt centralized algorithms for the scheduling mechanism. On the other hand, more recent research focuses on the integrated coverage and connectivity problem. This paper presented a MOGA with elitist selection, which yielded good results in terms of PF population. This framework can then be implemented in a more realistic model for the communication and sensing of the sensor networks. This paper present new method for improving connectivity using elitism with external population [SPEA] will provide to make clusters of networks. This method will decrease the distance between nodes that improves the coverage of wireless sensor networks.

\section{References}

[1] I.F. Akyildiz, W. Su, Y. Sankarasubramaniam, E. Cayirci, Wireless sensor networks: a survey, Computer Networks 38 (4) (2002) 393-422.

[2] B. Badrinath, J. Scholtz, M. Srivastava, K. Mills, V. Stanford, IEEE, IEEE Personal Communication, 2000 (Special issue on smart spaces and environments

[3] D. Li, K.D. Wong, Y.H. Hu, A.M. Sayeed, Detection, classification, and tracking of targets, IEEE Signal Processing Magazine 19 (2002) 17-29.

[4] P. Varshney, Distributed detection and data fusion, Springer-Verlag, 1996

[5] M. Cardei, J. Wu, Energy-efficient coverage problems in wireless ad-hoc sensor networks, Computer Communications 29 (2006) .413-420.

[6] S. Slijepcevic, M. Potkonjak, Power efficient organization of wireless sensor networks, in: Proceedings of the IEEE international conference on communications, vol. 2, 2001, pp. 472-476.

[7] C.C. Lai, C.K. Ting, R.S. Ko, An effective genetic algorithm to improve wireless sensor network lifetime for large-scale surveillance applications, in: Proceedings of the 2007 Congress on Evolutionary Computation, 2007, pp. 3531-3538

[8] DAMIEN JOURDAN, ,'Node placement for a wireless sensor network using a multi objective genetic algorithm.

[9] M. Cardei, D.Z. Du, Improving wireless sensor network lifetime through power aware organization, Wireless Networks 11 (3) (2005) 333-340

[10 ] Zhang. B.S. Lee, X.S. Wang, Aggregation in sensor networks with a user-provided quality of service goal, Information Sciences 178 (9) (2008) 2128-2149

[11] L. Krishnamachari, D. Estrin, S. Wicker, The impact of data aggregation in wireless sensor networks, in: Proceedings of the 22nd International Conference on Distributed Computing Systems Workshop, 2002, pp. 575-578, 2002

[12] W.R. Heinzelman, A. Chandrakasan, H. Balakrishnan, Energy-efficient communication protocol for wireless microsensor networks, in: Proceedings of the 33rd Annual Hawaii International Conference on System Sciences, 2000, pp. 1-10. 
[13] C. Ok, S. Lee, P. Mitra, S. Kumara, Distributed routing in wireless sensor networks using energy welfare metric, Information Sciences 180 (9) (2010) 1656-1670

[14] F. Marcelloni, M. Vecchio, Enabling energyefficient and lossy-aware data compression in wireless sensor networks by multi-objective evolutionary optimization, Information Sciences 180 (10) (2010) 1924-1941.

[15] S. Meguerdichian and M. Potkonjak, "Low power $0 / 1$ coverage and scheduling techniques in sensor networks," Tech. Rep. 030001, University of California at Los Angles, January 2003.

[16] D. Tian and N. D. Georganas, "A coveragepreserving node scheduling scheme for large wireless sensor networks," in WSNA '02: Proceedings of the 1st ACM international workshop on Wireless sensor networks and applications. New York, NY,USA: ACM, 2002, pp. 32-41.

[17] G. Xing, X. Wang, Y. Zhang, C. Lu, R. Pless , and C. Gill, "Integrated coverage and connectivity configuration for energy conservation in sensor networks," ACM Trans. Sen. Netw., vol. 1, no. 1, pp. 36-72, 2000

[18] F. Ye, G. Zhong, J. Cheng, S. Lu, and L. Zhang, "Peas: A robust energy conserving protocol for longlived sensor networks," in ICDCS '03: Proceedings of the 23rd International Conference on Distributed Computing Systems. Washington, DC, USA: IEEE Computer Society, 2003, p. 28

[19]. N. Chaiyarataiia and A. M. S. Zalzala, "Recent Developments in Evolutionary and Genetic Algorithms: Theory and Applications", Genetic Algorithms in Engineering Systems: Innovations and Applications, 24September 1997

[20] Zitzler E, Thiele L. Multi objective evolutionary algorithms: a comparative case study and the strength Pareto approach. IEEE Trans Evol Comput 1999;3(4):257-71.

[21] Deb K. Multi-objective optimization using evolutionary algorithms. New York: Wiley; 2001.

[22] Van Veldhuizen DA, Lamont GB. Multi objective evolutionary algorithms: analyzing the state-of-the-art. Evol Comput 2000;8(2): 125-47.

[23] Zitzler E, Thiele L. Multiobjective evolutionary algorithms: a comparative case study and the strength Pareto approach. IEEETrans Evol Comput 1999;3(4):257-71.

[24] Morse JN. Reducing the size of the nondominated set: pruning by clustering. Comput Oper Res 1980;7(12):55-66.

\section{BIOGRAPHY}

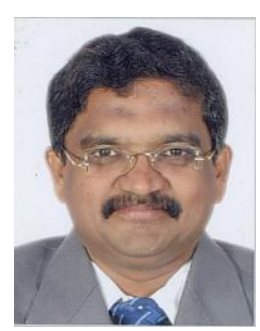

M.Siddappa received B.E degree in Computer Science \& Engineering from University of Mysore, Mysore, Karnataka, India in 1989, M.Tech from University of Mysore in 1993 and doctrol degree from Dr .MGR Educational Research Institute Chennai under supervision of Dr.A.S.Manjunatha, CEO, Manvish e-Tech Pvt. Ltd., Bangalore. He worked as project associate in IISc, Bangalore under Dr.M.P Srinivasan and Dr. V.Rajaraman from 1991 - 1993. He has teaching experience of 22 years and research of 5 years. He published 35 Technical Papers in National, International Conference and Journals. He is a member of IEEE and Life member of ISTE. He is working in the field of data structure and algorithms, Artificial Intelligence, Image processing and Computer networking. He worked as Assistant Professor in Department of Computer Science \& Engineering from 1996 to 2003 in Sri Siddhartha Institute of Technology, Tumkur. Presently, he is working as Professor and Head, Department of Computer Science \& Engineering from 1999 in Sri Siddhartha Institute of Technology, Tumkur. He has published nearly 31 papers in national and international journals. $\mathrm{He}$ has received best Engineering teacher award from ISTE for the year 2011.

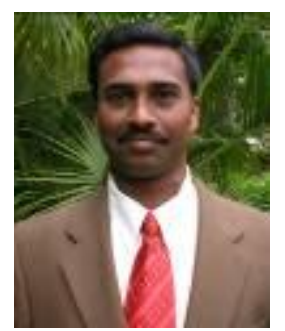

Mr. Channakrishna raju has 15 years of teaching experience for UG and PG courses in computer Science and Engg.,.and is presently working as Assistant Professor in the department of computer Science and Engg., in Sri Siddhartha Institute of Technology, Tumkur. He Obtained B.E from Bangalore University in the year 1995 and PG in software systems in the year 2000 from BITS Pilani. His research interests are in the areas of Wireless sensor networks and Artificial Intelligence.. Currently pursuing doctoral degree in Sri Siddhartha University, Tumkur, under the guidance of Dr. Siddappa M, Professor and head of the department of Computer Science and Engineering, Sri Siddartha Institute of Technology, Tumkur. 